to sit the illustrious Dalton, with his beautiful face, so like that of Newton, and the keenly intelligent Joule. I can give no other explanation than the fact of organic chemistry being then a new science that two pbilosophers of such eminence should come to the lectures of a mere tyro in science. I used to look upon them as two types of the highest progress in science. Newton had introduced law, order, and number into the movements of masses of matter in the universe; Dalton introduced the same into the minute masses which we call atoms; and Joule, with a keen insight into the operations and correlation of forces, connected them together, and showed their mutual equivalence.

I do not know whether these memories are of any use to you, but, such as they are, they are at your disposal for your lecture on the friend of my youth. Yours sincerely, LYON PLAYFAiR.

\section{WEIGHING BY A SERIES OF WEIGHTS.}

THIS subject, now under discussion in NATURE, is by no means new. The following remarks on the general theory of such questions may prove interesting. The problems are divisible into two classes :-

(I) To assign a series of weights so as to be able to weigh any weight of an integral number of pounds from $I$ to $n$ inclusive, the weights being placed in only one scale-pan.

(2) The same problem when the weights may be placed in both scale-pans.

Two other conditions may be imposed, viz. :-

(a) That no other weighings are to be possible.

(b) That each weighing is to be possible in only one way, i.e. to be unique.

The question considered in NATURE is of the second class, and is further subject to the two conditions above stated. Moreover, the problem for the number of pounds $\mathrm{I}, 4, \mathrm{r} 3,40, \& \mathrm{c} . \ldots$, as solved by the series of weights I; I, $3 ; \mathrm{I}, 3,9 ; \mathrm{I}, 3,9,27$ \&c...., respectively gives in each case the series containing the least number of weights for which the solution is possible.

For a number of pounds equal to 40 , the solution may be given by means of a single algebraic formula, constituting an identity, viz.

$\left(x^{-1}+\mathbf{I}+x\right)\left(x^{-3}+\mathrm{I}+x^{3}\right)\left(x^{-9}+\mathrm{I}+x^{9}\right)\left(x^{-27}+\mathbf{I}+x^{27}\right)$ $=x^{-40}+x^{-39}+\ldots \ldots+x^{-1}+\mathrm{r}+x+\ldots \ldots+x^{39}+x^{40}$.

This is easily verified. When the left-hand side is multiplied out, the resulting powers of $x$ are obtained by addition of certain indices, some positive and some negative. The indices in question are $1,3,9,27$, and, from the form of the factors, may in any single term be taken either positively or negatively. Thus one term on the left is $x^{-3} \cdot x^{9} \cdot x^{27}=x^{-3+9+27}$, giving the term $x^{33}$ on the right-hand side. The identity shows that every number from $I$ to 40 inclusive can be thus composed by the numbers $1,3,9,27$, taken positively or negatively. It shows, moreover, since the coefficients of the several powers on the right are each unity, that the composition is unique. The identity includes also negative integers from $-\mathrm{I}$ to -40 . This is necessitated by the very nature of the question.

The above is one out of eight distinct solutions of the problem before enunciated. Before giving the other seven, it will be as well to show the principles of solution in general.

Consider, first, the problem of the first class in which weights can only be placed in one scale-pan. The number of pounds to be weighed being $n$, we must discover the ways in which it is possible to break up the expression.

$$
\mathrm{I}+x+x^{2}+x^{3}+\ldots \ldots+x^{n}
$$

into similar expressions, for, as will be evident, each such factorization corresponds to a definite solution of the problem. By similar expression is to be understood one in which the coefficients are unity and the indices are in arithmetical progression.

At the outset may be remarked the trivial solution consisting of $n$ ones corresponding to the unfactorized expression. There is one solution of this kind in respect of every number $n$.

Putting the expression in the form

$$
\frac{\mathrm{I}-x^{n+1}}{\mathrm{I}-\mathrm{x}}
$$

NO. I IOI, VOL. 4.3 it will be clear that if $n+\mathbf{I}$ be a prime number, the expression cannot be factorized in the required form. This is a consequence of the properties of prime roots of unity.

Hence, when $n+\mathrm{I}$ is prime, the only solution is obtained by taking $n$ one-pound weights.

If, however, $n+$ I be not prime, the number of solutions depends upon the composite character of $n+\mathrm{I}$. If $n+\mathrm{I}=s t$ where $s$ and $t$ are primes, we may write

$$
\begin{gathered}
\frac{\mathbf{I}-x^{n+1}}{\mathbf{I}-x}=\frac{\mathbf{I}-x^{s t}}{\mathbf{I}-x}=\frac{\mathrm{I}-x^{s}}{\mathbf{I}-x} \cdot \frac{\mathbf{I}-x^{s t}}{\mathbf{I}-x} \\
=\left(\mathbf{I}+x+x^{2}+\ldots+x^{s-1}\right)\left(\mathbf{I}+x^{s}+x^{s s}+\ldots+x^{s t-s}\right),
\end{gathered}
$$

a factorization of the required form.

Multiplying $x^{p}$ in the first factor into $x^{q s}$ in the second, we find that the number $p+q s$ is composed by $p$ numbers equal to $I$, and $q$ numbers equal to $s$. In general, every number from I to $n$ inclusive can be composed by means of $s-I$ ones, and $t-I$ s's. That is to say, that all numbers of pounds from I to $n$ (where $n$ is of the form st $-\mathbf{I}$ ) can be weighed, and in a unique manner, by means of $s$ - I weights of I pound each, and $t-1$ weights of $s$ pounds each.

Another solution is obtained from the identity

$$
\frac{\mathrm{I}-x^{n+1}}{\mathrm{I}-x}=\frac{\mathrm{I}-x^{s t}}{\mathrm{I}-x}=\frac{\mathrm{I}-x^{t}}{\mathrm{I}-x} \cdot \frac{\mathrm{I}-x^{s t}}{\mathrm{I}-x_{t}}
$$

obtained from the former by interchange of $s$ and $t$. These two solutions, together with the trivial one, constitute the complete set of three solutions. For brevity, these may be represented in the form-

$$
\begin{aligned}
& \mathbf{I}^{s t-1}, \\
& \mathbf{I}^{s-1} s^{t-1}, \\
& \mathbf{I}^{t-1} t^{s-1},
\end{aligned}
$$

where $s^{t-1}$ means $t-\mathbf{I} s^{\prime}$ s, and so on.

From the above simple example it will be sufficiently clear that the number of solutions in the case of a given number $n$ is entirely dependent upon the form of $n+1$, when regarded as a product of prime factors. The number of solutions is not in all cases easy to determine, but some of the simpler results are in teresting. Suppose the number $n+r$ to be a product of $h$ unrepeated prime numbers, and, under these circumstances, represent the number of solutions in regard to the number $n$ by

$$
\left[\mathrm{I}^{k}\right] \text {. }
$$

Then it is easy to prove the relation-

$$
\begin{gathered}
{\left[\mathbf{I}^{k}\right]=\mathbf{I}+k\left[\mathrm{I}^{1}\right]+\frac{k(k-\mathbf{I})}{2 !}\left[\mathrm{I}^{2}\right]+\frac{k(k-\mathbf{I})(k-2)}{3 !}\left[\mathbf{I}^{3}\right]+\ldots} \\
\text { to } k \text { terms, }
\end{gathered}
$$

and thence to calculate the values of $\left[\mathrm{I}^{1}\right],\left[\mathrm{I}^{2}\right],\left[\mathrm{I}^{3}\right], \ldots$ in succession. The series of numbers thus obtained commences I, 3, I3, $75,54 \mathrm{I}, \ldots$ where it will be noticed that the second number, 3 , corresponds with the result obtained above for the case of $n+\mathbf{I}$, being of the form $s t$, or the product of two unrepeated primes. It may be observed that this series of numbers is of great interest, and presents itself frequently in mathematics, notably in Prof. Cayley's "Theory of the Analytical Forms called Trees" (see vol. xiii. and subsequent volumes of the Philosophical Magazine, and "Collected Papers," No. 203).

A much simpler case to consider is that for which $n+x$ is merely a power of a prime number. If $n+x$ be the kth power of a prime, represent the number of solutions in regard to the number $n$ by

A small amount of reffection shows the truth of the relation

whence

$$
[k]=2[k-1]
$$

$$
[k]=2^{k-1} \text {. }
$$

Turning now to the second class of problems, in which it is permissible to place weights in either or both scale-pans, it can be shown that the theory is not essentially different from that belonging to those of the second class. 
In order to weigh any number of pounds from $I$ to $n$ inclusive, we have to factorize the expression

$$
\begin{gathered}
x^{-n}+x^{(-n-1)}+x^{-(n-2)}+\ldots+n-1+\mathbf{1}+x+\ldots \\
+x^{n-2}+x^{n-1}+x^{n},
\end{gathered}
$$

which may be thrown into the form

$$
\frac{1-x^{2 n+1}}{x^{n}(1-x)}
$$

The solutions depend upon the composite character of the number $2 n+\mathrm{I}$. There always exists the trivial solution consisting of $n$ ones, and when $2 n+\mathrm{I}$ is prime, this constitutes the only solution.

Supposing $2 n+1$ the product of two primes, viz.

we may write

$$
2 n+\mathrm{I}=s t,
$$

$$
\begin{gathered}
\mathbf{1}-x^{2 n+1}=\frac{\mathbf{I}-x^{s t}}{x^{n}(\mathbf{I}-x)}=\frac{\mathbf{I}}{x^{n}(\mathbf{I}-x)} \cdot \frac{\mathbf{I}-x^{s}}{x^{n}} \cdot \frac{\mathbf{I}-x^{s t}}{\mathrm{I}-x^{s}} \\
=\frac{\mathrm{I}-x^{s}}{x^{\frac{s-1}{2}}(\mathbf{I}-x)} \cdot \frac{\mathbf{I}-x^{s t}}{x^{s t-1)}\left(1-x^{s}\right)} \\
=\left(x^{-\frac{s-1}{2}}+x^{-\frac{s-3}{2}}+\ldots+x^{-1}+\mathbf{1}+x+\ldots+x^{\left.\frac{s-3}{2}+x^{\frac{s-1}{2}}\right)}\right. \\
\times\left(x^{-\frac{s t-s}{2}}+x^{-\frac{s t-3 s}{2}}+\ldots+x^{-s}+\mathbf{I}+x^{s}+\ldots+x^{\frac{s t-3 s}{2}}+x^{\frac{s t-s}{2}}\right) .
\end{gathered}
$$

The factors appearing in this last expression are of the required form, and the factorization indicates that any number from $I$ to $n$ incl usive may be composed by means of $\frac{1}{2}(s-I)$ ones, and $\frac{1}{2}(t-I) s^{\prime} s$, if all the ones that are taken be taken either positively or negatively, and all the s's also either positively or negatively.

The solution may be represented, according to the method before explained, by

$$
\mathbf{I}^{\frac{2}{2}(s-1)} s^{\frac{1}{2}(t-1)},
$$

and the complete system of solutions will in the present case be denoted by

$$
\left\{\begin{array}{l}
\mathrm{I}^{\frac{1}{2}(s t-1)}, \\
\mathrm{I}^{\frac{1}{2}(s-1)} s^{\frac{1}{2}(t-1)}, \\
1^{\frac{1}{2}(t-1)} t^{\frac{1}{2}(s-1)} .
\end{array}\right.
$$

From the above, it is clear that there is a one-to-one correspond ence between the solutions of the second class problem in regard to the number $n$ and the solutions of the first class problem in regard to the number $2 n$. The theory of the second class problems is thus included in that of the first class problems. If we take the case considered in this journal of $n=40$ and both scale-pans, the number of solutions will be the same as that for $n=80$ and one scale-pan; the number depends upon the composite character of the integer $8 \mathrm{I}$, which is $3^{4}$; hence the number of solutions (see ante) is [4], which is $2^{3}$ or 8 .

Corresponding to the several identities-

$$
\begin{aligned}
& \text { I }-x^{81} \\
& x^{+10}(1-x) \\
& =x^{-49}+x^{-39}+\ldots+x^{-1}+\mathbf{1}+x+\ldots+x^{39}+x^{40} \text {; } \\
& \mathrm{I}-x^{81} \quad \mathrm{I}-x^{3} \\
& =\left(x^{-39}+x^{-36}+\ldots+x^{-3}+\mathrm{I}+x^{3}+\ldots+x^{36}+x^{39}\right)\left(x^{-1}+\mathrm{I}+x\right) ; \\
& \mathbf{I}-x^{81} \quad \mathbf{I}-x^{3} \\
& x^{36}\left(\mathbf{1}-x^{9}\right) \cdot x^{4}(\mathbf{I}-x) \\
& =\left(x^{-36}+x^{-27}+\ldots+\mathrm{I}+\ldots+x^{27}+x^{36}\right) \\
& \left(x^{-4}+x^{-3}+\ldots+\mathbf{I}+\ldots+x^{3}+x^{4}\right) \\
& \frac{\mathbf{I}-x^{81}}{x^{36}\left(\mathbf{I}-x^{3}\right)} \cdot \frac{\mathbf{I}-x^{9}}{x^{3}\left(\mathbf{I}-x^{3}\right)} \cdot \mathbf{I}-x^{3} \\
& =\left(x^{-36}+x^{-27}+\ldots+x^{36}\right)\left(x^{-3}+\mathbf{I}+x^{3}\right)\left(x^{-1}+\mathbf{I}+x\right) \text {; }
\end{aligned}
$$

NO. I IOI, VOL. 43 ]

$$
\begin{aligned}
& \frac{\mathbf{I}-x^{81}}{x^{27}\left(\mathbf{I}-x^{27}\right)} \cdot \frac{\mathbf{I}-x^{27}}{x^{13}(\mathbf{1}-x)} \\
& =\left(x^{-27}+\mathbf{I}+x^{27}\right)\left(x^{-13}+x^{-12}+\ldots+x^{13}\right) ; \\
& \frac{\mathbf{I}-x^{81}}{x^{-7}\left(\mathbf{1}-x^{27}\right)} \cdot \frac{\mathbf{1}-x^{27}}{x^{12}\left(\mathbf{1}-x^{3}\right)} \cdot \frac{\mathbf{1}-x^{3}}{x(\mathbf{1}-x)} \\
& =\left(x^{-: 7}+\mathrm{I}+x^{27}\right)\left(x^{-12}+x^{-9}+\ldots+x^{12}\right)\left(x^{-1}+\mathrm{I}+x\right) ; \\
& \frac{\mathbf{I}-x^{81}}{x^{27}\left(\mathrm{I}-x^{27}\right)} \cdot \frac{\mathbf{I}-x^{27}}{x^{9}\left(\mathbf{I}-x^{9}\right)} \cdot \frac{\mathbf{I}-x^{9}}{x^{4}(\mathbf{I}-x)} \\
& =\left(x^{-27}+\mathrm{I}+x^{27}\right)\left(x^{-9}+\mathrm{I}+x^{9}\right)\left(x^{-4}+x^{-3}+\ldots+x^{4}\right) ; \\
& \begin{array}{llll}
\mathbf{I}-x^{81} & \mathbf{I}-x^{27} & \mathbf{I}-x^{9} & \mathbf{I}-x^{3}
\end{array} \\
& x^{27}\left(\mathbf{I}-x^{77}\right) \cdot \frac{1}{x^{9}\left(1-x^{9}\right)} \cdot \frac{1}{x^{3}\left(1-x^{3}\right)} \cdot \frac{1-x^{3}}{x(1-x)} \\
& =\left(x^{-27}+\mathbf{I}+x^{27}\right)\left(x^{-9}+\mathbf{I}+x^{9}\right)\left(x^{-3}+\mathbf{I}+x^{3}\right)\left(x^{-1}+\mathbf{I}+x\right)
\end{aligned}
$$

These are the eight solutions represented by

$$
\begin{aligned}
& I^{40} \\
& I \cdot 3^{13} \\
& I^{4} \cdot 9^{4} \\
& I \cdot 3 \cdot 9^{4} \\
& I^{13} \cdot 27 \\
& I \cdot 3^{4} \cdot 27 \\
& I^{4} \cdot 9 \cdot 27 \\
& I \cdot 3 \cdot 9 \cdot 2 \%
\end{aligned}
$$

The subject is more fully entered into in a paper by myself in the Quarterly Fournal of Fure and Applied hicthematics for I886.

P. A. MACMAHON.

\section{THE SCIENTIFIC RESULTS OF THE}

\section{OCCUPATION OF BRITISH NEW GUTNEA.}

$A$ VOLUMINOUS and extremely interesting report on the first year of the administration of British New Guinea by Sir William MacGregor was issued some time ago by the Colonial Office. It deals with the period ending June 30, 1889. One of the sections of the report deals with "scientific results," which we are glad to notice have a place like "finance," "legislation," "trade and shipping," and the other usual divisions of these colonial reports. In sending the report home, Sir Henry Norman, the Governor of Queensland, observes that it is fortunate that the administrator is most anxious to obtain the best scientific results on his visits and tours, and that he is well able to judge for himself in such matters. The scientific collections, therefore, are made with judgment, and the various reports on collections are of interest and value. Sir William MacGregor himself, in summing up the scientific results, says that during the year some addition was made to our knowledge of the natural history of the country. Unfortunately, it is not possible to set out fully the progress made, as the report on specimens sent to England had not reached him at the moment of writing. It is his hope, however, that in future all specimens collected may be examined in Australia, so that the information gained can be kept together and be summarized in each annual Report.

Geology.-Thirty-one small bags of specimens were examined by Mr. Jack, Government Geologist of Queensland. All except two were from the Louisiade and D'Entrecasteaux groups. Mr. Jack's report, which will be found to be interesting and valuable, is given in an appendix. A set of specimens, covering the route from Manu-Manu to the summit of the Owen Stanley Range, was submitted to careful examination by Mr. Rands, Assistant Government Geologist of Queensland, who at the same time classified the specimens collected in the Rigo district. Mr. Rand's report will also be found in an appendix. Although not forming any part of the work of the year, there is added to the same appendix a report on certain geological specimens collected by Mr. C. S. Wilkinson. These three reports practically contain all that is really known of the geology of the country.

Ornithology. - The greater portion of the birds obtained were classified by Mr. de Vis. His report, prepared after much careful labour, is added as an appendix. From it may be inferred that the probability is that no great addition will be made now to the more beautiful and gorgeous birds of British New Guinea. 